\title{
Correction to: Keeping pace with forestry: Multi-scale conservation in a changing production forest matrix
}

\author{
Adam Felton (1), Therese Löfroth, Per Angelstam, Lena Gustafsson, \\ Joakim Hjältén, Annika M. Felton, Per Simonsson, Anders Dahlberg, \\ Matts Lindbladh, Johan Svensson, Urban Nilsson, Isak Lodin, \\ P. O. Hedwall, Anna Sténs, Tomas Lämås, Jörg Brunet, \\ Christer Kalén, Bengt Kriström, Pelle Gemmel, Thomas Ranius
}

Published online: 16 November 2019

\section{Correction to: Ambio}

https://doi.org/10.1007/s13280-019-01248-0

In the original published article, the sentence "Nevertheless, semi-natural forest remnants continue to be harvested and fragmented (Svensson et al. 2018; Jonsson et al. 2019), and over 2000 forest-associated species (of 15000 assessed) are listed as threatened on Sweden's red-list, largely represented by macro-fungi, beetles, lichens and butterflies (Sandström 2015)."under the section Introduction was incorrect.

The correct version of the sentence is "Nevertheless, semi-natural forest remnants continue to be harvested and fragmented (Svensson et al. 2018; Jonsson et al. 2019), and approximately 2000 forest-associated species (of 15000 assessed) are on Sweden's red-list, largely represented by macro-fungi, beetles, lichens and butterflies (Sandström 2015).”

Open Access This article is distributed under the terms of the Creative Commons Attribution 4.0 International License (http:// creativecommons.org/licenses/by/4.0/), which permits unrestricted use, distribution, and reproduction in any medium, provided you give appropriate credit to the original author(s) and the source, provide a link to the Creative Commons license, and indicate if changes were made.

Publisher's Note Springer Nature remains neutral with regard to jurisdictional claims in published maps and institutional affiliations.

\author{
Adam Felton ( $\square)$ \\ Address: Southern Swedish Forest Research Centre, SLU, Box 49, \\ Rörsjöv 1, 23053 Alnarp, Sweden. \\ e-mail: adam.felton@slu.se
}

The original article can be found online at https://doi.org/10.1007/ s13280-019-01248-0.
Therese Löfroth

Address: Southern Swedish Forest Research Centre, SLU, Box 49, Rörsjöv 1, 23053 Alnarp, Sweden.

e-mail: therese.lofroth@slu.se

\section{Per Angelstam}

Address: Faculty of Forest Sciences, School for Forest Management, Swedish University of Agricultural Sciences, PO Box 43, 73091 Skinnskatteberg, Sweden.

e-mail: per.angelstam@slu.se

\section{Lena Gustafsson}

Address: Department of Ecology, Swedish University of Agricultural Sciences, P.O. Box 7044, 75007 Uppsala, Sweden.

e-mail: lena.gustafsson@slu.se

\section{Joakim Hjältén}

Address: Department of Wildlife, Fish, and Environmental Studies, Swedish University of Agricultural Sciences, 90183 Umeå, Sweden. e-mail: joakim.hjalten@slu.se

\section{Annika M. Felton}

Address: Southern Swedish Forest Research Centre, SLU, Box 49, Rörsjöv 1, 23053 Alnarp, Sweden.

e-mail: annika.felton@slu.se

\section{Per Simonsson}

Address: Härnösand, Sweden.

e-mail: per.simon@outlook.com

\section{Anders Dahlberg}

Address: Department of Forest Mycology and Plant Pathology, Swedish University of Agricultural Sciences, PO Box 7026, 75007 Uppsala, Sweden.

e-mail: anders.dahlberg@slu.se

\section{Matts Lindbladh}

Address: Southern Swedish Forest Research Centre, SLU, Box 49, Rörsjöv 1, 23053 Alnarp, Sweden.

e-mail: matts.lindbladh@slu.se

\section{Johan Svensson}

Address: Department of Wildlife, Fish, and Environmental Studies, Swedish University of Agricultural Sciences, 90183 Umeå, Sweden. e-mail: johan.svensson@slu.se 


\section{Urban Nilsson}

Address: Southern Swedish Forest Research Centre, SLU, Box 49, Rörsjöv 1, 23053 Alnarp, Sweden.

e-mail: urban.nilsson@slu.se

\section{Isak Lodin}

Address: Southern Swedish Forest Research Centre, SLU, Box 49, Rörsjöv 1, 23053 Alnarp, Sweden.

e-mail: isak.lodin@slu.se

\section{P. O. Hedwall}

Address: Southern Swedish Forest Research Centre, SLU, Box 49, Rörsjöv 1, 23053 Alnarp, Sweden.

e-mail: per-ola.hedwall@slu.se

\section{Anna Sténs}

Address: Department of Historical, Philosophical and Religious Studies, Umeå University, 90187 Umeå, Sweden.

e-mail: anna.stens@umu.se

\section{Tomas Lämås}

Address: Department of Forest Resource Management, Swedish University of Agricultural Sciences, 90183 Umeå, Sweden.

e-mail: tomas.lamas@slu.se

\section{Jörg Brunet}

Address: Southern Swedish Forest Research Centre, SLU, Box 49, Rörsjöv 1, 23053 Alnarp, Sweden.

e-mail: jorg.brunet@slu.se

\section{Christer Kalén}

Address: National Forest Agency, Bryggargatan 19-21, 50338 Borås, Sweden.

e-mail: christer.kalen@skogsstyrelsen.se

\section{Bengt Kriström}

Address: Department of Forest Economics, Swedish University of Agricultural Sciences, 90183 Umeå, Sweden.

e-mail: Bengt.kristrom@slu.se

\section{Pelle Gemmel}

Address: Southern Swedish Forest Research Centre, SLU, Box 49, Rörsjöv 1, 23053 Alnarp, Sweden.

e-mail: pelle.gemmel@slu.se

\section{Thomas Ranius}

Address: Department of Ecology, Swedish University of Agricultural Sciences, P.O. Box 7044, 75007 Uppsala, Sweden.

e-mail: thomas.ranius@slu.se 